\title{
ANÁLISIS DE LA DEMANDA DE CHOCOLATE QUESADA EN DIFERENTES SECTORES DE LA ZONA SUR DE BOGOTÁ
}

\section{ANALYSIS OF THE DEMAND FOR QUESADA CHOCOLATE IN DIFFERENT SECTORS OF THE SOUTHERN AREA OF BOGOTÁ}

\section{DOI: www.doi.org/10.54198/innova08.06}

Salome del Pilar López-Peña ${ }^{1}$

Johan Stiven Morales Aguilar ${ }^{2}$

(iD) Sergio Camilo Rojas González ${ }^{3}$

Cómo citar esté artículo: López Peña, S. P., Morales Aguilar, J.S., \& Rojas González, S.C. Aanálisis de la demanda de chocolate quesada en diferentes sectores de la zona sur de Bogotá, Revista Innova ITFIP, 8 (1), 68-75

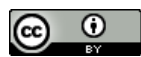

Recibido: Febrero de 2021.Aprobado: Mayo de 2021

\section{Resumen}

Con el presente estudio de investigación, se pretendió obtener información relevante acerca del comportamiento de los consumidores de la empresa CASA LUKER en la zona sur de la ciudad de Bogotá, una vez de identificó una disminución significativa en la demanda de Chocolate Quesada durante dos periodos consecutivos. En ese sentido se propuso un estudio que permitió analizar las causas de la disminución de las ventas del chocolate Quesada en la zona sur de Bogotá, específicamente las localidades de Bosa y Tunjuelito.

\footnotetext{
${ }^{1}$ Magíster en Salud Pública, Investigadora Universidad Minuto de Dios UNIMINUTO, Grupo de investigación GICABS, Semillero Aplicación de las Ciencias Económicas y Administrativas para el Desarrollo (ACEAD), ORCID https://orcid.org/0000-0002-10437543, splopezp@unal.edu.co, Colombia.

2 estudiante de Administración de Empresas, Universidad Minuto de Dios UNIMINUTO, Grupo de investigación GICABS, Semillero Aplicación de las Ciencias Económicas y Administrativas para el Desarrollo (ACEAD), ORCID https://orcid.org/00000003-1028-5700, Johan.morales-ag@uniminuto.edu.co, Colombia.

3 vinculado a la Corporación Universitaria Minuto De Dios, estudiante de Administración de Empresas, Universidad Minuto de Dios UNIMINUTO, Grupo de investigación GICABS, Semillero Aplicación de las Ciencias Económicas y Administrativas para el Desarrollo (ACEAD), ORCID https://orcid.org/0000-0001-5515-526X, sergio.rojas@ uniminuto.edu.co, Colombia.
} 
REVISTA INNOVA ITFIP, 8 (1). 68-75. JUN. 2021

Se presenta un estudio mixto y transversal de estudio de caso, definido sobre la demanda de Chocolate Quesada en dos tiendas de la zona sur de Bogotá. Este trabajo de campo se realizó en dos fases. Por último, se realizó un análisis de las encuestas realizadas en los dos sectores cuya población tiene el mismo estrato socioeconómico y características similares, generando así las posibles soluciones que permita una mayor rotación del chocolate Quesada.

Cabe resaltar el hecho de que la mayoría de personas encuestadas fue de sexo femenino, con un $71 \%$, el estrato 2 fue el que más encuestados tuvo con un $70 \%$ seguido del estrato 1 con un $17 \%$, así mismo el rango de edad al que se le realizaron la mayor cantidad de encuestas fue de 20 - 39 años. El 83\% de la población encuestada afirmó reconocer el chocolate Quesada y, de estos, solo a un $32 \%$ manifestó no gustarle la nueva presentación.

En general el comportamiento de estos estratos está encaminado a una relación de un precio medio con un buen sabor de chocolate y con un consumo un poco bajo, en donde sale a relucir el estrato 1, siendo este el único que tiene como presencia el precio más alto del mercado y sin ningún encuestado que prefiera el precio más bajo, a esto se adiciona el hecho de que su consumo sea un poco mayor al de los otros estratos analizados, concluyendo así que la mayoría de personas prefieren un consumo moderado y un precio medio para el chocolate.

Palabras clave: Demanda de chocolate, chocolate en barra, Bogotá

\begin{abstract}
This research study was intended to obtain relevant information about the behavior of consumers of the company CASA LUKER in the southern area of the city of Bogotá, once it identified a significant decrease in the demand for Chocolate Quesada during two consecutive periods. A study was proposed to analyze the causes of the decrease in sales of Quesada chocolate in the southern zone of Bogotá, specifically the towns of Bosa and Tunjuelito.

A mixed and transversal case study is presented, defined on the demand for Chocolate Quesada in two stores in the southern zone of Bogotá. This fieldwork was carried out in two phases. Finally, an analysis was made of the surveys carried out in the two sectors whose population has the same socioeconomic stratum and similar characteristics, thus generating the possible solutions that would allow a greater rotation of Quesada chocolate.

It is important to highlight the fact that the majority of people surveyed were women, with $71 \%$, stratum 2 had the most respondents with $70 \%$, followed by stratum 1 with $17 \%$, and the age range in which most surveys were conducted was 20-39 years. $83 \%$ of the population surveyed said they recognized Quesada chocolate and, of these, only 32\% said they did not like the new presentation.
\end{abstract}


In general, the behavior of these strata is directed towards a relationship of an average price with a good chocolate flavor and with slightly low consumption, where the first stratum comes out, is this the only one that has as presence the highest price of the market and without any respondent that prefers the lowest price, to this is added the fact that its consumption is a little higher than the other strata analyzed, concluding that most people prefer a moderate consumption and an average price for chocolate.

Keywords: Demand for chocolate, chocolate bars, Bogotá

\section{Introducción}

Con el presente estudio de investigación, se pretendió obtener información relevante acerca del comportamiento de los consumidores de la empresa CASA LUKER en la zona sur de la ciudad de Bogotá, una vez de identificó una disminución significativa en la demanda de Chocolate Quesada durante dos periodos consecutivos. En ese sentido se propuso un estudio de mercado que permitió identificar los consumidores que actualmente están consumiendo los productos de la empresa anteriormente mencionada.

\section{Materiales y Métodos}

Se presenta un estudio mixto y transversal de estudio de caso, definido sobre la demanda de Chocolate Quesada en dos tiendas de la zona sur de Bogotá. Este trabajo de campo se realizó en dos fases. La primera fase se realizó en el Mercado Hogar Tunjuelito, de la localidad de Tunjuelito, donde se aplicó una encuesta que buscaba dar respuesta a la disminución de ventas del chocolate Quesada en Bogotá. La segunda fase, con cierta reconstrucción a las preguntas iniciales, se realizó en la localidad de Bosa específicamente en los supermercados Metropol, consolidando aspectos como el género de los consumidores, la edad, el estrato socioeconómico entre otros aspectos que le brindan a la investigación un margen de análisis más amplio.

Por último, se realizó un análisis de las encuestas realizadas en los dos sectores cuya población tiene el mismo estrato socioeconómico y características similares, generando así las posibles soluciones que permita una mayor rotación del chocolate Quesada. 
REVISTA INNOVA ITFIP, 8 (1). 68-75. JUN. 2021

\section{Marco teórico}

Un estudio de campo realizado por estudiantes de posgrados de psicología de la fundación universitaria Konrad Lorenz, el cual toma una muestra de 40 familias en los estrados 2 y 3 de la ciudad de Bogotá, con el fin de identificar el grado de fidelidad de las personas a una marca o producto, determinando como ellos se hicieron fieles a la marca en especial a que es lo que más compran en cantidad y el número de veces de la recompra. Los resultados que arrojó la investigación fueron diversos en el sentido que da luz a los procesos investigativos sobre el campo, resaltando hechos como los patrones de la compra donde los clientes en gran parte de los casos saben lo que van a llevar y sus marcas favoritas (Sandoval, 2009).

El anterior estudio arroja que en varias ocasiones los consumidores finales tienen su marca favorita, y no suelen prestar atención al precio del chocolate, y de la misma forma se genera la recompra del mismo. Es por ello por lo que se hace notar la importancia del departamento de marketing porque son ellos los que generan a través de sus campañas crear expectativa al shopper y así lograr los objetivos propuestos y poder impactar al consumidor final.

Por otra parte, hay que considerar otro análisis de campo, el cual va a evaluar los siguientes aspectos. Analizar por qué el consumidor compra una marca en vez de otra, identificar la participación en el mercado de las marcas líderes y los seguidores dentro del mercado, realizar una comparación entre las marcas para identificar que las diferencias, por último, ejecutar una prueba de marketing en el mercado y como este interfiere en el momento de la compra. Este estudio arrojó que "el marketing es sin duda un motor importante en la industria que este es el que ayuda a estabilizar a un producto como líder dentro un determinado sector del mercado, es allí donde las herramientas que este provee a una marca o varias dentro un determinado sector del mercado" (Gabrielli, 2016)

Se concluye que el consumo del cliente se basa en la fidelización a una marca antigua y que ha sido líder en el mercado por mucho tiempo, esto no quiere decir que el mercado se cierre y solo se prefiera esta, las demás marcas tienen que estar impulsadas por el sector de marketing para poder llegar a acercarse al principal competidor o líder.

Marcela Alfaro Jaramillo, hace una comparación con los habitantes de Francia en cuanto al consumo de Chocolate y saca la siguiente conclusión: "Afinidad o Presupuesto: Los colombianos y los franceses gastan la misma cantidad de dinero a la semana en la compra de chocolate. Es decir, entre $\$ 0$ y $\$ 7.500$ o lo equivalente en euros entre $0 €$ y $3 €$ aproximadamente por semana. Teniendo en cuenta la teoría, esté anterior resultado llama bastante la atención. Puesto que estamos afirmando que en un país emergente como Colombia (con un PIB de 366 billones de US para el 2012)" (Jaramillo, 2013). 
También cabe resaltar que en ambos países las tabletas son las presentaciones de chocolate más populares. Seguramente son las preferidas por su practicidad y porque se adaptan a todo tipo de ocasión. El chocolate en bebida también es popular al igual que la panadería de chocolate (pero ésta última en menor proporción)" (Jaramillo, 2013).

Los resultados anteriores están relacionados con las Teorías Culturales, ya que según las tradiciones de cada país se presenta mayor predilección hacia ciertas presentaciones de chocolate. Por ejemplo, en Colombia es tradicional consumir chocolate como bebida mientras que en Francia es más común consumirlo como panadería o Momentos de consumo: En ambos países se consume chocolate, cotidianamente en las comidas principales. Dicho resultado está relacionado con las presentaciones de chocolate, siendo muy popular para el desayuno en Colombia la bebida de chocolate y en Francia la panadería de chocolate (Pan de Chocolate).

Los cambios en el mundo empresarial son constantes y es allí donde las empresas, tienen que adaptarse a dichos cambios, los cuales son de suma importancia para mantener a una empresa a flote.

"El mundo empresarial del cacao en el país deberá enfrentar con otra visión organizacional estos desafíos; son necesarios cambios positivos y enraizados en cada individuo y actor de este producto. Es necesaria la conservación de espacios de análisis de las capacidades de la materia prima, los productores que deben posicionarse del mercado sólidamente con derivados de alta calidad. Por lo tanto, a través de esta investigación se analizará las generalidades de la cultura organizacional desde la perspectiva de los diferentes autores, posteriormente se describirá la industria de cacao y chocolate ecuatoriano para finalmente contrastar los enfoques del cambio organizacional en ciertas empresas del País, A través de una revisión de autores, y una compilación bibliográfica que contraste las diferentes perspectivas de cambio que el sector requiere.” (Lombeida, 2019).

Es así como se plantea la necesidad de reactivar los mercados y dinamizar el mercado frente a los nuevos retos que presentan, como consumidores, determinado la capacidad de análisis de las partes. El chocolate del país goza una gran aceptación internacional donde este se puede concentrar en el mercado extranjero usando el continente europeo como el foco de trabajo arduo para el cacao colombiano debido a que este continente tiene gran cantidad de países y una extensión por metro cuadrados corta a comparación de otros países.

Finalmente, es importante mencionar que el mercado colombiano cacaotero está siendo cada vez más afectados por la competencia desleal y la venta de la materia prima como lo es el caco para la preparación del chocolate a bajos precios a nivel interno, debido a la devaluación del peso colombiano y la competencia desleal en el mercado interno. 


\section{Resultados y Discusión}

Cabe resaltar el hecho de que la mayoría de personas encuestadas fue de sexo femenino, con un $71 \%$, el estrato 2 fue el que más encuestados tuvo con un $70 \%$ seguido del estrato 1 con un $17 \%$, así mismo el rango de edad al que se le realizaron la mayor cantidad de encuestas fue de 20 - 39

años. El 83\% de la población encuestada afirmó reconocer el chocolate Quesada y, de estos, solo a un $32 \%$ manifestó no gustarle la nueva presentación.

En el estrato 1 se prefiere un consumo de 1 a 2 veces por semana, y también están dispuestos a pagar el mayor precio de chocolate en el mercado. Una de las posibles causas al pago de un mayor valor del producto se relaciona con el bajo consumo que lleva a preferir que este sea de mayor agrado y mayor calidad.

El estrato 2 tiene un comportamiento similar al estrato 1, sin embargo, la diferencia entre estos dos radica en el precio que están dispuestos a pagar por el chocolate, siendo así que el estrato 2, el 56\% están dispuestos a pagar de 4.200 a 4.400 siendo estos los precios medios del mercado.

En el estrato 3 se encuentra un comportamiento bastante moderado a comparación de los otros dos estratos, teniendo en cuenta que 57\% de los encuestado consumen chocolate solo 1 a 2 veces por semana y prefirieren un precio que oscile entre 4.200 y 4.400, siendo así un estrato con un comportamiento un poco similar al estrato 2 y bastante diferente al estrato 1 .

\section{Discusión}

El consumo interno en un país como Colombia de productos propios como lo es el cacao, ha tenido a lo largo de la historia diversas tendencias de consumo, es decir consumirlo en dulces, desayunos o puro. En este momento el mercado presenta una tendencia de consumo hacia productos que décadas atrás no se pensarían como lo son los modificadores en polvo, es decir el chocolate en polvo, los productos reducidos en azúcar o sin azúcar. Esto ha permito que el mercado tenga que cambiar y la producción del cacao también.

A lo anterior se suma que, muchos de los participantes de la categoría de cacao son productores que rinden el cacao que se produce con diversos productos químicos. Lo cual afecta la calidad del producto, pero a su vez este le permite tener un precio menor en el mercado a los productores que utilizan más licor de cacao en la producción de sus productos.

Este hecho conlleva a que muchas de los compradores potenciales se guíen por el precio del producto, en una economía en recesión y con crisis económica latente, dejando de lado la tendencia de consumo por las marcas líderes y pasando a una mayor acogida de las marcas propias o económicas en varios segmentos del mercado. 
Cabe resaltar que el mercado es cambiante y volátil, esto genera que las empresas están en constante innovación y estrategias agresivas para poder rescatar a la productos y marcas de quedar relegados de estas nuevas formas de consumo, es allí donde se plantea la necesidad de explorar para la marca nuevas formas de presentación, precio y muestreo en las diferentes plataformas para poder alcanzar un nuevo punto de venta. Mejor que los presentados hasta el momento.

\section{Conclusiones}

En general el comportamiento de estos estratos está encaminado a una relación de un precio medio con un buen sabor de chocolate y con un consumo un poco bajo, en donde sale a relucir el estrato 1, siendo este el único que tiene como presencia el precio más alto del mercado y sin ningún encuestado que prefiera el precio más bajo, a esto se adiciona el hecho de que su consumo sea un poco mayor al de los otros estratos analizados, concluyendo así que la mayoría de personas prefieren un consumo moderado y un precio medio para el chocolate.

Tomando como punto de partida el hecho de que el sabor favorito de chocolate para la gente es el tradicional con un 54\% sobre los otros sabores y que su consumo es de 1 a 2 veces por semana en un $54 \%$ y de 2 a 4 veces por semana un $46 \%$, esto también se ve influenciado por el valor del producto, en donde, por lo general, se prefieren precios de 4.200 a 4.400, siendo este precio el más acogido por las personas.

Para concluir el consumo de chocolate en barra en los últimos tiempos, han sufrido drásticos cambios debido a que las nuevas generaciones de shopper buscan nuevas experiencias de consumo saludable, lo cual afecta la tendencia del mercado. A lo anterior, se suma que el consumidor final se está rigiendo por los precios del mercado, que algunas veces están elevados y otras están por debajo; dependiendo de la categoría y el abastecimiento que haya en el momento.

\section{Referencias}

Efraín, G. R. (2014). INDUSTRIA SOCIEDAD Y ARTE. BOGOTÁ.

G, B. (2018). Chocolate, politics, and Peacebuilding. San José De Apartadó: Springer.

Gabrielli, V. y. (2016). online brand community the integrated marketing communication system: When chocolate becomes seductive like a person. journal of marketing, 385-402.

Huamachuco de la cuba, O. (2017). Cacao, producción, consumo y comercio del periodo prehispánico a la actualidad en América Latina. Fronteras de la Historia, 237-242. 
J, C. (1978). REMINICENCIAS DE SANTAFÉ DE BOGOTÁ. BOGOTÁ: INSTITUTO COLOMBIANO DE CULTURA.

L, j. c. (2019). Plan De Mercado Internacional Para El Chocolate Premium Colombiano. Bachelor's thesis. Mercadeo y Negocios Internacionales.

LOMBEIDA, M. D. (2019). El cambio organizacional en la comercialización de chocolate ecuatoriano. Gestión Joven, 7-9.

LONDOÑO. (2002). EL ARTE Y LA PUBLICIDAD EN LA NACIONAL DE CHOCOLATES 1920-1960. MEDELLIN: NACIONAL DE CHOCOLATES.

M, A. J. (2013). Caracterización del consumo de chocolate en el caso Colombia y Francia. Universidad EIA.

Nuttall, C. \&. (1994). Comercialización de chocolate y otros aspectos de la industria confitera en todo el mundo. Fabricación y uso industrial del chocolate. Springer, Boston MA, 362-385.

Sandoval, R. R. (2009). Patrones de elección de marca. Revista Latinoamericana de Psicología, 3 22.

Superintendencia de industria y comercio. (2012). Estudio sobre el sector cacaotero. Bogotá: Licencia Creative Commons Atribución-No Comercial Sin Derivadas 2.5 Colombia. 